\title{
Fast Secure Comparison for Medium-Sized Integers and Its Application in Binarized Neural Networks
}

\author{
Mark Abspoel ${ }^{1,2}$, Niek J. Bouman ${ }^{3}$, \\ Berry Schoenmakers ${ }^{3}$, and Niels de Vreede $^{3}$ \\ ${ }^{1}$ Centrum Wiskunde \& Informatica (CWI), Amsterdam \\ 2 Philips Research Eindhoven \\ 3 Technische Universiteit Eindhoven
}

\begin{abstract}
In 1994, Feige, Kilian, and Naor proposed a simple protocol for secure 3 -way comparison of integers $a$ and $b$ from the range $[0,2]$. Their observation is that for $p=7$, the Legendre symbol $(x \mid p)$ coincides with the sign of $x$ for $x=a-b \in[-2,2]$, thus reducing secure comparison to secure evaluation of the Legendre symbol. More recently, in 2011, $\mathrm{Yu}$ generalized this idea to handle secure comparisons for integers from substantially larger ranges $[0, d]$, essentially by searching for primes for which the Legendre symbol coincides with the sign function on $[-d, d]$. In this paper, we present new comparison protocols based on the Legendre symbol that additionally employ some form of error correction. We relax the prime search by requiring that the Legendre symbol encodes the sign function in a noisy fashion only. Practically, we use the majority vote over a window of $2 k+1$ adjacent Legendre symbols, for small positive integers $k$. Our technique significantly increases the comparison range: e.g., for a modulus of 60 bits, $d$ increases by a factor of 2.9 (for $k=1$ ) and 5.4 (for $k=2$ ) respectively. We give a practical method to find primes with suitable noisy encodings.

We demonstrate the practical relevance of our comparison protocol by applying it in a secure neural network classifier for the MNIST dataset. Concretely, we discuss a secure multiparty computation based on the binarized multi-layer perceptron of Hubara et al., using our comparison for the second and third layers.
\end{abstract}

\section{Introduction}

Secure integer comparison has been a primitive of particular interest since the inception of multiparty computation (MPC). In 1982, even before general multiparty computation had been realized, Yao introduced the millionaires' problem, in which two millionaires want to determine who of them is richer without revealing any information beyond the outcome of this comparison to each other or to any third party [21]. Secure comparison has been investigated extensively since. A whole range of solutions is available with every solution aiming for a particular trade-off. Nonetheless, for arithmetic MPC schemes - as opposed to MPC 
schemes for Boolean circuits - secure comparison remains among the most expensive basic operations. Hence, for applications that require many comparisons, achieving high throughput (important for privacy-preserving data processing applications) or low latency (crucial for certain applications, like blind auctions for real-time advertisement sales) can be challenging.

\section{$1.1 \quad$ Related Work}

Whereas most secure comparison protocols work over finite fields of arbitrary order, $\mathrm{Yu}[22]$ presents a comparison protocol that only works for specifically chosen prime moduli. Although this clearly poses a restriction in terms of applicability, the main benefit is that the specifically chosen prime modulus $p$ enables $\mathrm{Yu}$ to perform a comparison in a single round of communication in the online phase (the offline preprocessing phase requires three communication rounds), albeit in a range that is small compared to $p$ (see Section 3.5 for explicit bounds). Namely, he chooses $p$ such that the pattern of quadratic residues and non-residues modulo $p$ coincides with the sign function on a given interval symmetric around zero, which is an idea that goes back to a protocol due to Feige, Kilian, and Naor [3], who use it to compute the sign of an element $x \in[-2,2]$ in $\mathbb{Z} / 7 \mathbb{Z}$. Yu's comparison protocol for comparing arbitrary elements $a, b \in \mathbb{Z} / p \mathbb{Z}$ essentially works by breaking up the full-range comparison into several medium-range comparisons of the above type by performing a digit decomposition.

\subsection{This Paper}

In this paper, we pursue the line of work initiated by Yu [22]. Our main contribution is that we achieve an improvement in the comparison range while keeping the bit length of the prime modulus fixed. Concretely, we propose a protocol that, for a fixed prime-length, achieves roughly a three-fold increase of the comparison range (over Yu's results), while still enjoying a single-round online phase, at the cost of a constant amount of additional communication and some additional local computations. Also, we present a two-online-rounds protocol that achieves roughly a five-fold increase in the comparison range when compared to Yu's approach. In other words, to compare two integers that lie in a given range (symmetric around zero), our methods require a smaller prime than the prime required for the protocol from [22]. Keeping the finite-field modulus as small as possible or within the machine's word size could be important, for example, in a setting where MPC protocols run on constrained hardware platforms. On such platforms, the complexity of prime-field arithmetic (which is directly related to the prime size) can have a significant impact on the runtime performance. Our protocols can be found in Section 5 .

The main idea is to somewhat relax the constraints on the prime modulus $p$ : instead of requiring that the Legendre symbols of all elements in the interval $[-d, d]$, for a given positive integer $d$, coincide with the sign function, we only require this coincidence for most elements (in a specific sense). Let us, for some fixed prime $p$, say that there is an error at position $x \in[-d, d]$ if $(x \mid p) \neq \operatorname{sgn}(x)$. 
Our improvement is based on exploiting a "local redundancy" property enjoyed by the sign function that lets us correct such errors as long as they are sufficiently "sparse", by means of inspecting also the Legendre symbols of some neighboring positions and then performing a majority vote.

This new approach raises the question of how to find primes that give rise to increased ranges. In Section 4, we present some results that considerably simplify this search, including tables of suitable primes for various bit lengths, and leave as an open problem to prove asymptotic lower and upper bounds on the growth of the comparison range in the prime's bit length.

\subsection{Application: Efficient Neural Network Evaluation in MPC}

To demonstrate the practical value of our work, we apply our new comparison protocol to the problem of securely evaluating a neural network, in which the sign function is used as non-linearity. We use a binarized multi-layer perceptron (BMLP) for recognizing handwritten digits, as described in [7], which is trained (in the clear) on the well-known MNIST handwritten-digits data set. We consider an MPC scenario in which the input images are secret-shared between the parties, which then securely evaluate the BMLP to obtain the estimated digit in secret-shared form.

\section{Preliminaries}

Integer Intervals. Whenever we write $[a, b]$, unless stated otherwise, we mean the integer interval $\{a, \ldots, b\} \subset \mathbb{Z}$, which is empty if $a>b$. The half-open interval $(a, b]$ is defined as $[a+1, b]$.

Arithmetic Black Box. We suppose that we are given a secure arithmetic blackbox $(\mathrm{ABB})$ functionality that can securely evaluate multiplication and linear forms over the finite field $\mathbb{Z} / p \mathbb{Z}$. We write $\llbracket x \rrbracket$ for the value $x \in \mathbb{Z} / p \mathbb{Z}$ encrypted under the ABB (e.g., $x$ is secret-shared among a set of parties, or perhaps encrypted under some homomorphic encryption scheme). Abusing notation, for small $x \in \mathbb{Z} / p \mathbb{Z}$ we will also refer to $x$ as an integer in $\mathbb{Z}$, given as the canonical lift of the residue class modulo $p$ to the integers $\left[-\left\lfloor\frac{p}{2}\right\rfloor,\left\lfloor\frac{p}{2}\right\rfloor\right]$.

Sign vs. Binary Sign. The sign function and the binary sign function are respectively defined as

$$
\operatorname{sgn}(z)=\left\{\begin{array}{rl}
1 & \text { if } z>0, \\
0 & \text { if } z=0, \\
-1 & \text { if } z<0
\end{array} \quad \operatorname{bsgn}(z)=\left\{\begin{aligned}
1 & \text { if } z \geq 0 \\
-1 & \text { if } z<0
\end{aligned}\right.\right.
$$

Comparing two integers $a$ and $b$ is achieved by evaluating the sign (or bsgn) of their difference $a-b$. The sgn function gives rise to a three-way comparison, while the bsgn function corresponds to two-way comparison. In this paper, we 
will start our analysis in terms of the sgn function, but for reasons that will become clear later our protocols evaluate the bsgn function (i.e., achieve twoway comparison). We will sometimes be a bit sloppy and use the word "sign" also for the bsgn function; the precise meaning should nonetheless still be clear from its context.

The Legendre symbol. Recall that for any odd prime $p$ and any integer $a$, the Legendre symbol is defined as

$$
(a \mid p)=\left\{\begin{aligned}
0 & \text { if } a \equiv 0 \quad(\bmod p) \\
1 & \text { if } a \text { is a quadratic residue modulo } p \\
-1 & \text { otherwise. }
\end{aligned}\right.
$$

The Legendre symbol is a completely multiplicative function, which means that $(a \mid p)(b \mid p)=(a b \mid p)$ for all $a, b \in \mathbb{Z}$, and for any integer $a$ the value of $(a \mid p)$ only depends on $[a]_{p}$, the residue class of $a$ modulo $p$. The identity $(a \mid p) \equiv a^{\frac{p-1}{2}}$ $(\bmod p)$ is known as Euler's criterion. The law of quadratic reciprocity asserts that for odd primes $p$ and $q$,

$$
(p \mid q)(q \mid p)=(-1)^{\frac{p-1}{2} \frac{q-1}{2}} .
$$

Securely evaluating Legendre symbols. In principle, we can securely evaluate the Legendre symbol via Euler's criterion, which would require $O(\log p)$ secure multiplications. The complete multiplicativity of the Legendre symbol enables the following constant-rounds protocol for securely evaluating the Legendre symbol in the preprocessing model with an single-round online phase. In the preprocessing phase, we generate a secret-shared pair $(\llbracket r \rrbracket, \llbracket(r \mid p) \rrbracket)$ of a random non-zero $r$ together with its Legendre symbol. In the online (input-dependent) phase, we securely multiply $\llbracket a \rrbracket \cdot \llbracket r \rrbracket$, open the result and then compute

$$
\llbracket(a \mid p) \rrbracket=(a r \mid p) \llbracket(r \mid p) \rrbracket .
$$

Note that the security of the protocol requires that $a \not \equiv 0(\bmod p)$, which should be taken into account when using this protocol.

Blum primes. A prime $p$ for which $p \equiv 3(\bmod 4)$ is called a Blum prime. By Euler's criterion, -1 is a quadratic non-residue modulo $p$ if and only if $p$ is a Blum prime. Hence, for any Blum prime $p$, the map $x \mapsto(x \mid p)$ is an odd function for $x \in[-\lfloor p / 2\rfloor,\lfloor p / 2\rfloor]$ (which follows immediately from the multiplicativity of the Legendre symbol), i.e., it enjoys the same symmetry around the origin as the sign function.

\section{Evaluating the Sign Function using Legendre Symbols}

\subsection{Redundancy Property of the Sign Function}

In this section we show that the sign function enjoys a "local redundancy" property, which lets us correct sign-flip errors by means of majority-decoding as long as those errors occur sparsely (in a sense defined below). 
Definition 1. Let $k \geq 0$ be an integer, and let $\mathcal{T}=\left[t_{1}, t_{2}\right]$ be an interval of integers with $t_{2}-t_{1} \geq 2 k$. We say that a function $e: \mathcal{T} \rightarrow\{0,1\}$ is an error function on $\mathcal{T}$ admissible for $k$ if $e(x)=0$ for all $x \in[-(k+1), k+1] \cap \mathcal{T}$ and if $\sum_{i=-k}^{k} e(y+i) \leq k$ holds for all $y \in\left[t_{1}+k, t_{2}-k\right]$.

Lemma 1. Let $k$ and $\mathcal{T}$ be as in Definition 1, and let e be an error function on $\mathcal{T}$ admissible for $k$. Then,

$$
\operatorname{sgn}\left(\sum_{i=-k}^{k}(-1)^{e(x+i)} \operatorname{sgn}(x+i)\right)=\operatorname{sgn}(x)
$$

holds for all $x \in\left[t_{1}+k, t_{2}-k\right]$.

The proof will clarify why we require in Definition 1 that an admissible error function $e(x)$ has an "error-free" region around $x=0$; informally speaking, the reason is that the sign function undergoes its sign change at $x=0$, which means that there is "less room" for errors under majority-decoding in this region.

Proof. We will prove the statement for $\mathcal{T}=[-a, a]$ where $a \geq k$ is any integer. This implies the claim for any subinterval of $\mathcal{T}$ of cardinality at least $2 k+1$. Note that because of symmetry (in the sign function as well as in the definition of an admissible error function), it suffices to prove the statement for $x \geq 0$. We distinguish three cases for $x$. If $x=0$, we have $\sum_{i=-k}^{k}(-1)^{e(i)} \operatorname{sgn}(i)=\sum_{i=-k}^{k} \operatorname{sgn}(i)=$ $\operatorname{sgn}(x)=0$, where the first equality follows because $e$ is admissible for $k$ and the second equality follows from the fact that summing an odd function over an interval symmetric around zero gives the value zero.

Second, if $x>k$, we have

$$
\sum_{i=-k}^{k}(-1)^{e(x+i)} \operatorname{sgn}(x+i)=\sum_{i=-k}^{k}(-1)^{e(x+i)}>0,
$$

where the equality follows because $\operatorname{sgn}(x+i)=1$ for all $i \in[-k, k]$ and the inequality follows because $e$ is admissible for $k$.

For the third (and final) case, suppose that $x \in[1, k]$. We have

$$
\begin{aligned}
\sum_{i=-k}^{k}(-1)^{e(x+i)} \operatorname{sgn}(x+i) & =\sum_{i=-k}^{k-x+1} \operatorname{sgn}(x+i)+\sum_{i^{\prime}=k-x+2}^{k}(-1)^{e\left(x+i^{\prime}\right)} \operatorname{sgn}\left(x+i^{\prime}\right) \\
& =\sum_{j=x-k}^{k+1} \operatorname{sgn}(j)+\sum_{j^{\prime}=k+2}^{k+x}(-1)^{e\left(j^{\prime}\right)} \operatorname{sgn}\left(j^{\prime}\right) \\
& =1+x+\sum_{j^{\prime}=k+2}^{k+x}(-1)^{e\left(j^{\prime}\right)} \\
& \geq(1+x)+(1-x)=2
\end{aligned}
$$




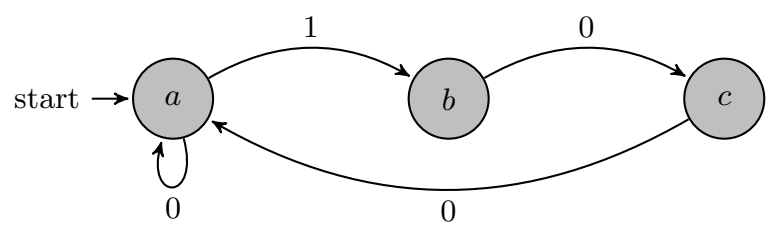

Fig. 1: Finite state machine generating bit strings with at most one 1 in every three consecutive bits.

\subsection{Counting Admissible Error Functions}

How many admissible error functions can there be on a given set $\mathcal{T}$ and for a given integer $k$ ? In this section, we will give an upper bound for $k=1$. To prove the upper bound, we will need the following lemma, which is well known.

Lemma 2 ("Volume Bound for Hamming Balls"). Let $\mathcal{S}_{n}$ be the set of all bit sequences of length $n$ produced using by concatenating bits from a binary source $X$. Then, it holds that

$$
2^{H(X) n-o(n)} \leq\left|\mathcal{S}_{n}\right| \leq 2^{H(X) n}
$$

where $H(X)$ denotes the Shannon entropy of $X$.

In Figure 1, we show a finite state machine (FSM) that defines a language of bit strings such that every string in the language has the following property: for every window of three consecutive bit positions, at most one position will be " 1 ". If we impose a probability distribution on the outgoing edges of state (a), then we can view the FSM as a random binary source, which lets us compute the entropy which we need to apply Lemma 2.

Proposition 1. Let $\mathcal{T}:=[-t, t]$ with $t \in \mathbb{N}$ such that $t \geq 2$. Let $\mathcal{E}_{1}$ be the set of all error functions on $\mathcal{T}$ admissible for $k=1$. Then,

$$
\left|\mathcal{E}_{1}\right| \leq 2^{t-2} \text {. }
$$

Proof. For all error functions $e \in \mathcal{E}_{1}$, it holds by definition that $e(x)=0$ for $x \in[-2,2]$. We may choose the function values on remaining positions, that is, the intervals $[3, t]$ and $[-t,-3]$, freely under the constraint that $e(x-1)+$ $e(x)+e(x+1) \leq 1$ for all $x \in[-t+1, t-1]$. In each such interval, there are, according to Lemma $2, N \leq 2^{\lambda(t-2)}$ choices, with $\lambda:=H(X)$, the entropy rate of the FSM in Figure 1. It is easy to see that $H(X)=1 / 2$ : namely, the binary decision in state $a$ corresponds to one bit of entropy but produces on average two output bits (either the length-1 output " 0 " or the length-3 output sequence "100"). Because the choices for the two intervals are independent, in total there are $N^{2}$ choices for $e$, hence $\left|\mathcal{E}_{1}\right| \leq 2^{t-2}$. 


\subsection{Avoiding Zero by Restricting to Odd Positions}

As mentioned in the preliminaries, if we use the single-online-round protocol for securely evaluating the Legendre symbol, we may not evaluate the Legendre symbol on the zero element. A simple trick to avoid zero (also used in [22]) is to restrict to evaluation of odd inputs by using the map $x \mapsto 2 x+1$. Note that this implies that we cannot compute $\operatorname{sgn}(x)$ using the single-online-round protocol; instead we will evaluate bsgn $(x)$. Removing the conditions on the Legendre symbols at even positions gives rise to the following definition.

Definition 3. Let $k$ be a non-negative integer, and let $p>2 k+1$ be a Blum prime. We define $d_{k}^{*}(p)$ as the largest integer $d$ such that for all integers $x$ with $1 \leq x \leq d$ it holds that

$$
\sum_{i=-k}^{k}(2(x+i)+1 \mid p)>0
$$

and we set $d_{k}^{*}(p)=0$ if no such $d$ exists.

Note that for any Blum prime $p$ for which $d_{0}(p)>1$ (which implies that $d_{0}(p)$ is even), it is easy to see that it holds that $d_{0}^{*}(p)=\frac{1}{2} d_{0}(p)-1$. For $k>0$, such simple relations do not seem to exist. This means, for example, that a prime $p$ that gives rise to a high value for $d_{1}(p)$, does not necessarily give a high value for $d_{1}^{*}(p)$, and vice versa.

\subsection{Bounds on $d_{0}(p)$}

The value $d_{0}(p)$ can be interpreted as the position just before the appearance of the first quadratic non-residue. Let $n_{1}(p)$ denote the smallest quadratic nonresidue. Finding bounds on $n_{1}(p)$ is a well-known problem in number theory, with important contributions from Polyà, Vinogradov and Burgess, among others. The best explicit upper bound that is currently known (for $p$ a Blum prime) is due to Treviño [19]:

$$
d_{0}(p)+1=n_{1}(p) \leq 1.1 \sqrt[4]{p} \log p .
$$

Graham and Ringrose [5] proved an unconditional asymptotic lower bound (improving on a previous result by, independently, ${ }^{4}$ Fridlender [4] and Salié [14]), namely, that there exist infinitely many primes for which

$$
d_{0}(p)+1=n_{1}(p) \geq c \cdot \log (p) \cdot \log \log \log p .
$$

for some absolute constant $c .^{5}$

Lamzouri et al. [10] prove that conditional on the Generalized Riemann Hypothesis, for all primes $p \geq 5$ it holds that

$$
d_{0}(p)+1=n_{1}(p)<(\log p)^{2} .
$$

\footnotetext{
${ }^{4}$ Ankeny [2] attributes this result to Chowla, but does not provide a reference.

${ }^{5}$ In the literature, this is also written as $n_{1}(p)=\Omega(\log (p) \cdot \log \log \log p)$, where $\Omega$ is Hardy-Littlewood's Big Omega: $f(n)=\Omega(g(n)) \Longleftrightarrow \limsup _{n \rightarrow \infty}|f(n) / g(n)|>0$.
} 


\subsection{Bounds on $d_{1}(p)$}

Hudson [8] proves an upper bound on the least pair of quadratic non-residues. Formally, let $n_{2}(p)$ be the smallest value such that $n_{2}(p)$ and $n_{2}(p)+1$ are quadratic non-residues. For $k=1$, it must hold that $d_{1}(p)<n_{2}(p)$, because an "error pattern" consisting of two consecutive quadratic non-residues (such that $\left.n_{2}(p) \in[1,(p-3) / 2]\right)$ cannot be corrected using a majority vote in a window of length $2 k+1=3$. Hudson's bound is as follows. For every $p \geq 5$ we have that

$$
d_{1}(p)<n_{2}(p) \leq\left(n_{1}(p)-1\right) q_{2},
$$

where $q_{2}$ is the second smallest prime that is a quadratic non-residue modulo $p$.

Hildebrand [6] proves another upper bound on $n_{2}(p)$ : for every $\epsilon>0$ there exists a constant $p_{0}$ such that for all $p \geq p_{0}$,

$$
d_{1}(p)<n_{2}(p) \leq p^{1 /(4 \sqrt{e})+\epsilon} .
$$

Sun [17] gives a construction for generating all elements $n$ in $\mathbb{Z} / p \mathbb{Z}$ such that $n$ and $n+1$ are quadratic non-residues.

Lemma 3 ([17]). Let $p$ be an odd prime and let $g$ be a primitive root of $p$. Then,

$$
\begin{aligned}
\mathcal{U} & :=\{n \in \mathbb{Z} / p \mathbb{Z} \mid(n \mid p)=(n+1 \mid p)=-1\} \\
& =\left\{u_{k} \in \mathbb{Z} / p \mathbb{Z} \mid u_{k} \equiv \frac{\left(g^{2 k-1}-1\right)^{2}}{4 g^{2 k-1}} \bmod p, \quad k=1, \ldots,\left\lfloor\frac{p-1}{4}\right\rfloor\right\}
\end{aligned}
$$

We can interpret this lemma as giving a collection of upper bounds on $d_{1}(p)$, that is, $d_{1}(p)<n_{2}(p) \leq u_{k}$ holds for every $k=1, \ldots,\lfloor(p-1) / 4\rfloor$.

An error pattern that consists of two quadratic non-residues that are separated by one arbitrary position can also not be corrected using a majority vote in a window of length $2 k+1=3$. Inspired by Sun, we prove the following lemma.

Lemma 4. Let $p$ be a Blum prime, let $b=((2 \mid p)+1) / 2 \in\{0,1\}$ and let $g$ be a primitive root of $p$. Then,

$$
\begin{aligned}
\mathcal{V} & :=\{n \in \mathbb{Z} / p \mathbb{Z} \mid(n \mid p)=(n+2 \mid p)=-1\} \\
& =\left\{v_{k} \in \mathbb{Z} / p \mathbb{Z} \mid v_{k} \equiv \frac{\left(g^{2 k-b}-1\right)^{2}}{2 g^{2 k-b}} \bmod p, \quad k=1, \ldots,(p-3) / 4\right\} .
\end{aligned}
$$

Also this lemma can be viewed as giving a collection of upper bounds on $d_{1}(p)$. If $(n \mid p)=(n+2 \mid p)=-1$, then a decoding error (under majority decoding with $k=1$ ) will occur at position $n+1$, hence we have that $d_{1}(p) \leq v_{k}$ holds (instead of strict inequality) for every $k=1, \ldots,(p-3) / 4$. 
Proof. Let $\chi(x)=(x \mid p)$ for all $x \in \mathbb{Z} / p \mathbb{Z}$. Jacobsthal [9] proves that for $p$ a Blum prime,

$$
\begin{aligned}
& |\{n \in \mathbb{Z} / p \mathbb{Z} \mid \chi(n)=\chi(n+2)=-1 \wedge \chi(n+1)=1\}|=\frac{p-1+2(2 \mid p)}{8} \\
& \text { and }|\{n \in \mathbb{Z} / p \mathbb{Z} \mid \chi(n)=\chi(n+1)=\chi(n+2)=-1\}|=\frac{p-5-2(2 \mid p)}{8}
\end{aligned}
$$

Hence, by summing the cardinalities of the above sets, we get that

$$
|\{n \in \mathbb{Z} / p \mathbb{Z} \mid \chi(n)=\chi(n+2)=-1\}|=\frac{p-3}{4} .
$$

For $j=1,2, \ldots,(p-3) / 2$, let $r_{j} \equiv\left(g^{j}-1\right)^{2} /\left(2 g^{j}\right) \bmod p$. Then, $r_{j}+2 \equiv$ $\left(g^{j}+1\right)^{2} /\left(2 g^{j}\right) \bmod p$. It now follows that $\chi\left(r_{j}\right)=\chi\left(r_{j}+2\right)=(-1)^{j} \chi(2)$ for all $j=1,2, \ldots,(p-3) / 2$. Hence, $\chi\left(r_{2 k-(\chi(2)+1) / 2}\right)=\chi\left(r_{2 k-(\chi(2)+1) / 2}+2\right)=-1$ for all $k=1,2, \ldots,(p-3) / 4$.

It remains to prove that $r_{s} \neq r_{t} \bmod p$ for all $s, t \in[1,(p-3) / 2]$ with $t \neq s$; for this part we can re-use Sun's proof technique used in the proof of Lemma 3. Namely, for all $s, t \in[1,(p-3) / 2]$ with $t \neq s$, we have that $g^{s+t} \not \equiv 1 \bmod p$ (since $g$ is a primitive root), which implies that $g^{s}-g^{t} \not \equiv\left(g^{s}-g^{t}\right) / g^{s+t} \bmod p$. Hence, $g^{s}+g^{-s} \not \equiv g^{t}+g^{-t} \bmod p$ from which we obtain that $r_{s} \not \equiv r_{t} \bmod p$. We can now conclude that

$$
\{n \in \mathbb{Z} / p \mathbb{Z} \mid \chi(n)=\chi(n+2)=-1\}=\left\{r_{2 k-b} \in \mathbb{Z} / p \mathbb{Z} \mid k \in[1,(p-3) / 4]\right\},
$$

and the claim follows.

\section{Finding a Prime for a Given $k$-Range}

In order to find a prime that, for given integers $k$ and $D_{k}$, gives rise to $d_{k}(p) \geq$ $D_{k}$, we could in principle take a naive approach by letting a computer exhaustively enumerate the primes in increasing order and compute the Legendre symbols at $a=1, \ldots, D_{k}$, and stop when they are all 1 . Although this approach works for small values of $k$ and $D_{k}$ (say for $D_{1}<200$ ), for larger $D_{k}$ this will become intractable.

We can speed up the calculation of $d_{k}$ by using the multiplicativity of the Legendre symbol, the law of quadratic reciprocity and the Chinese Remainder Theorem (CRT). Moreover, we may speed up the computation by enumerating over values $p$ that already satisfy some conditions on the Legendre symbols, using a wheel data structure $[13,16]$. We will first review the problem for the case $k=0$ and then extend the method to the case $k=1$. Our approach also works for arbitrary $k$, and we supply the relevant extensions, but we note that its practicality rapidly diminishes as $k$ increases. 


\subsection{Finding Primes with High $d_{0}(p)$}

Recall that finding a prime $p^{\prime}$ such that $d_{0}\left(p^{\prime}\right) \geq D$ means that $p^{\prime}$ must be a Blum prime such that the elements $1, \ldots, D$ are quadratic residues modulo $p^{\prime}$. By the complete multiplicativity of the Legendre symbol, it suffices to find a Blum prime $p$ such that all primes $q \leq D$ are quadratic residues modulo $p$.

Proposition 2. Let $q$ be an odd prime, and $p$ a Blum prime. Then, it holds that

$$
(q \mid p)=(-p \mid q) \text {. }
$$

Proof. It holds that $(q \mid p)=(p \mid q)^{-1}(-1)^{\frac{p-1}{2} \frac{q-1}{2}}=(p \mid q)(-1)^{\frac{q-1}{2}}=$ $(p \mid q)(-1 \mid q)=(-p \mid q)$, where the first equality holds by the law of quadratic reciprocity, the second holds because $p$ is a Blum prime, the third follows from Euler's criterion and the fourth follows from the multiplicativity property of the Legendre symbol.

Let $\mathcal{R}_{q}=\left\{[r]_{q}:(-r \mid q)=1\right\}$. Then, $q$ is a quadratic residue modulo $p$ if and only if

$$
[p]_{q} \in \mathcal{R}_{q}
$$

This represents an (exclusive) disjunction of linear congruences:

$$
p \equiv r_{1}(\bmod q) \vee \ldots \vee p \equiv r_{\ell}(\bmod q),
$$

where $\mathcal{R}_{q}=\left\{\left[r_{1}\right]_{q}, \ldots,\left[r_{\ell}\right]_{q}\right\}$.

Let $q_{1}, \ldots, q_{m}$ denote all odd primes that are in $[1, D]$. The condition that all integers $[1, D]$ are quadratic residues modulo $x$ thus gives rise to the following system of simultaneous disjunctions of linear congruences:

$$
x \equiv 7(\bmod 8), \quad[x]_{q_{1}} \in \mathcal{R}_{q_{1}}, \ldots, \quad[x]_{q_{m}} \in \mathcal{R}_{q_{m}},
$$

where the first congruence ensures that $(-1 \mid x)=-1$ and $(2 \mid x)=1$.

Suppose for each $i=1, \ldots, m$ we choose a residue class $\left[a_{i}\right]_{q_{i}} \in \mathcal{R}_{q_{i}}$, and we consider the resulting vector $\left(\left[a_{1}\right]_{q_{1}}, \ldots,\left[a_{m}\right]_{q_{m}}\right)$. We may choose the $\left[a_{i}\right]_{q_{i}}$ independently since the $q_{i}$ are distinct primes. An element $\left(\left[a_{1}\right]_{q_{1}}, \ldots,\left[a_{m}\right]_{q_{m}}\right) \in$ $\mathcal{R}_{q_{1}} \times \cdots \times \mathcal{R}_{q_{m}}=: \mathcal{R}$ is in one-to-one correspondence with an arithmetic progression of step $Q$ of solutions to the above system of congruences, that is, $x, x+Q, x+2 Q, \ldots$ where $Q=8 \prod_{i \in[m]} q_{i}$. Linnik's theorem [11] (combined with Xylouris' bound [20]) asserts that there will be a prime in this arithmetic progression whose size is bounded as $O\left(Q^{5}\right)$.

Finding the smallest such prime. Finding some prime that satisfies the above system is relatively easy, since we may fix a vector $\left(\left[a_{1}\right]_{q_{1}}, \ldots,\left[a_{m}\right]_{q_{m}}\right) \in \mathcal{R}$. We can then enumerate all positive integers $x$ such that $[x]_{q_{i}}=\left[a_{i}\right]_{q_{i}}$ via the constructive proof of the CRT, and output the first solution that is prime. However, finding the smallest prime that satisfies the above system is a (much) harder task, as it involves searching over the full set $\mathcal{R}$, whose cardinality is exponential in $m$. 
In practice, we may simply enumerate all integers $x$ in ascending order, and check whether $x$ satisfies the system of Equation (5) rather than computing the Legendre symbols at $1, \ldots, D_{0}$ explicitly. We can speed up the computation by precomputing the sets $\mathcal{R}_{q_{i}}$ and storing them in memory. We can check many congruences at once by combining sets of congruences using the CRT. For example, for moduli $q, q^{\prime}$ we have that $[x]_{q} \in \mathcal{R}_{q}$ and $[x]_{q^{\prime}} \in \mathcal{R}_{q^{\prime}}$ if and only if $[x]_{q q^{\prime}} \in \mathcal{R}_{q q^{\prime}}$,

$$
\mathcal{R}_{q q^{\prime}}:=\left(\mathcal{R}_{q}+\{0, q, \ldots,(\ell / q-1) q\}\right) \cap\left(\mathcal{R}_{q^{\prime}}+\left\{0, q^{\prime}, \ldots,\left(\ell / q^{\prime}-1\right) q^{\prime}\right\}\right),
$$

where $\ell=\operatorname{lcm}\left(q, q^{\prime}\right)$ and ' + ' denotes Minkowski addition. Note that we have abused notation here slightly, and represented the sets $\mathcal{R}_{m}$ for each modulus $m$ as the set of integers in $[0, m-1]$ that are the canonical lifts of the residue classes mod $m$. By recursion, the above extends to combining more than two sets of congruences.

\subsection{Finding Primes with High $d_{1}(p)$}

For $k>0$, for $d_{k}(p) \geq D$ to hold for some positive integer $D$, it is no longer necessary that $p$ satisfies each disjunction of congruences in Equation (5); instead, some subsets suffice. For example, for $d_{1}(p) \geq 6$ we need $(2 \mid p)=1$ and at least one of $(5 \mid p)=1$ or $(6 \mid p)=(2 \mid p)(3 \mid p)=(3 \mid p)=1$, otherwise Equation (2) fails to hold for $a=5$.

In order for Equation (2) to hold, we have one set of congruences for every length- $(2 k+1)$ subinterval of $[-k, d]$; even for $k=1$ this quickly grows prohibitively large for non-trivial lower bounds $D$ on $d_{k}(p)$. While for $k>0$ the density of primes $p$ satisfying $d_{k}(p) \geq D$ is greater than for $k=0$, the search becomes a lot more expensive.

For $k=1$, we simplify our search for $p$ with $d_{1}(p) \geq D_{1}$ with an extra condition: we also require $d_{0}(p) \geq D_{0}$ where $D_{1} \leq\left(D_{0}\right)^{2}$. This ensures that each integer in $\left(D_{0}, D_{1}\right]$ has at most one prime factor greater or equal to $D_{1}$. Under this restriction, we get a condition equivalent to $d_{1}(p) \geq D_{1}$ which requires fewer computations to check.

Definition 4. Let $D_{0}, D_{1}$ be non-negative integers with $D_{0}<D_{1} \leq\left(D_{0}\right)^{2}$. Let $q, q^{\prime}$ be distinct primes. We say that $\left\{q, q^{\prime}\right\}$ is a related pair on $\left(D_{0}, D_{1}\right]$ if $D_{0}<$ $q, q^{\prime} \leq D_{1}$ and there exist positive integers $x, y<D_{0}$ such that $\left|x q-y q^{\prime}\right| \leq 2$ and $\max \left\{x q, y q^{\prime}\right\} \leq D_{1}$.

Proposition 3. Let $D_{0}, D_{1}$ be non-negative integers with $D_{0}<D_{1} \leq\left(D_{0}\right)^{2}$, and let $p$ be a Blum prime with $d_{0}(p) \geq D_{0}$. Then $d_{1}(p) \geq D_{1}-1$ if and only if the following condition holds: for every related pair of primes $\left\{q, q^{\prime}\right\}$ on $\left(D_{0}, D_{1}\right]$ it holds that $(q \mid p)=1 \vee\left(q^{\prime} \mid p\right)=1$.

Proof. Let $a$ be any positive integer such that $a \leq D_{1}$. First, we show that $(a \mid p)=-1$ if and only if $a$ has a prime factor $q>D_{0}$ and $(q \mid p)=-1$. Suppose $a$ has a prime factor $q>D_{0}$ with $(q \mid p)=-1$. Since $\frac{a}{q}<\frac{a}{D_{0}} \leq \frac{D_{1}}{D_{0}} \leq D_{0}$, we 
have $(a / q \mid p)=1$, hence $(a \mid p)=-1$. If $a$ does not have a prime factor $q>D_{0}$ with $(q \mid p)=-1$, then taking any prime factor $q^{\prime} \mid a$, it must hold that $q^{\prime}>D_{0}$, in which case $\left(q^{\prime} \mid p\right)=1$ by assumption, or $q^{\prime} \leq D_{0}$, in which case $\left(q^{\prime} \mid p\right)=1$ by $d_{0}(p) \geq D_{0}$.

We now finish the proof by showing $d_{1}(p)<D_{1}-1$ if and only if there is some related pair $q, q^{\prime}$ such that $(q \mid p)=\left(q^{\prime} \mid p\right)=-1$. We have $d_{1}(p)<$ $D_{1}-1$ if and only if there exists an integer $x$ such that $1<x \leq D_{1}-1$ and $(x-1 \mid p)+(x \mid p)+(x+1 \mid p)<0$. This latter inequality holds if and only if at least two of $\{x-1, x, x+1\}$ have Legendre symbol -1 . By the above, this holds if and only if two of these numbers have respective prime factors $q, q^{\prime}>D_{0}$ and $(q \mid p)=\left(q^{\prime} \mid p\right)=-1$. For these $q, q^{\prime}$, we have that they constitute a related pair, since they each have a multiple in $\{x-1, x, x+1\}$ and $x+1 \leq D_{1}$. Conversely, for any related pair there exists such an interval $\{x-1, x, x+1\}$ with $1<x \leq D_{1}-1$.

Proposition 3 gives sufficient conditions for $d_{1}(p)>D_{1}-1$ in terms of related pairs of primes that have to satisfy certain disjunctions of congruences. If we want to include those disjunctions in a system as shown in Equation (5), we need to represent them in the same form. For every pair of related primes $\left\{q, q^{\prime}\right\}$, the condition that $(q \mid p)=1 \vee\left(q^{\prime} \mid p\right)=1$ in Proposition 3 corresponds to taking the union of the associated residue sets $\mathcal{R}_{q}$ and $\mathcal{R}_{q}^{\prime}$ of the related primes $q$ and $q^{\prime}$. That is, let $\ell=\operatorname{lcm}\left(q, q^{\prime}\right)$, then

$$
\mathcal{R}_{q, q^{\prime}}:=\left(\mathcal{R}_{q}+\{0, q, \ldots,(\ell / q-1) q\}\right) \cup\left(\mathcal{R}_{q}^{\prime}+\left\{0, q^{\prime}, \ldots,\left(\ell / q^{\prime}-1\right) q^{\prime}\right\}\right),
$$

where ' + ' denotes Minkowski addition, and again we abuse notation and canonically lift residue classes modulo $m$ to the integers $[0, m-1]$. We can now express the related-primes disjunction of congruences as

$$
[x]_{\ell} \in \mathcal{R}_{q, q^{\prime}} .
$$

Since this disjunction of congruences has exactly the same form as the other disjunctions in Equation (5) we can also take intersections (using Equation (6)) between a related-primes congruence $\mathcal{R}_{q, q^{\prime}}$ and another disjunction of congruences.

\subsection{Finding Primes with High $d_{k}(p)$}

Definition 5. Let $D_{0}, D_{k}$ be non-negative integers with $D_{0}<D_{k} \leq\left(D_{0}\right)^{2}$. Let $Q=\left\{q_{0}, \ldots, q_{k}\right\}$ be a set of $k+1$ distinct primes. We say that $Q$ is a related set on $\left(D_{0}, D_{k}\right]$ if $Q \subseteq\left(D_{0}, D_{k}\right]$ and there exist positive integers $x_{0}, \ldots, x_{k}<D_{0}$ such that:

1. for any $i$ with $0 \leq i \leq k$ we have $x_{i} q_{i} \leq D_{k}$

2. for any $i, j$ with $0 \leq i<j \leq k$ it holds that $\left|x_{i} q_{i}-x_{j} q_{j}\right| \leq 2 k$

Proposition 4. Let $D_{0}, D_{k}$ be non-negative integers with $D_{0}<D_{k} \leq\left(D_{0}\right)^{2}$, and let $p$ be a Blum prime with $d_{0}(p) \geq D_{0}$. Then $d_{k}(p) \geq D_{k}-k$ if and only if the following condition holds: for every set $Q$ of $k+1$ distinct primes related on $\left(D_{0}, D_{k}\right]$, it holds that there exists some $q \in Q$ with $(q \mid p)=1$. 


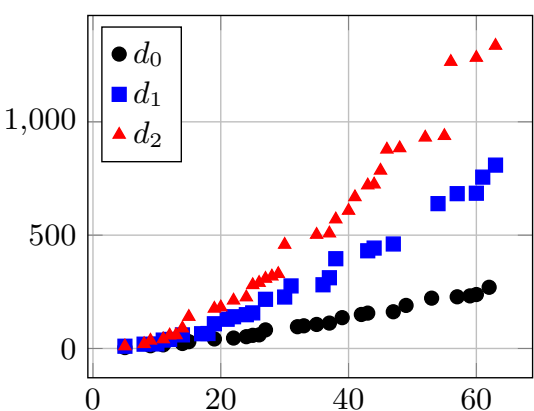

bit length of prime $(\ell)$

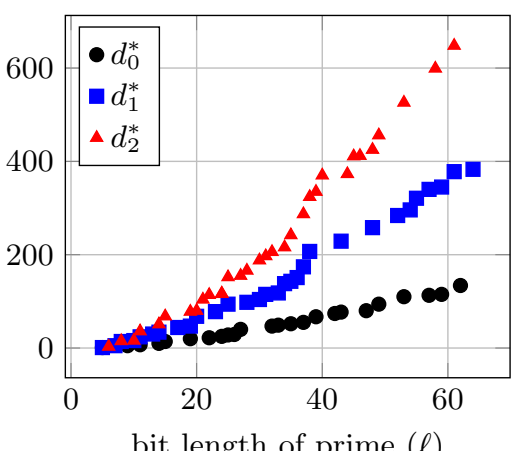

bit length of prime $(\ell)$

Fig. 2: Graphical comparison of the comparison range achieved by Yu's method $\left(d_{0}\right.$ and $\left.d_{0}^{*}\right)$ vs. our method. The data for $d_{0}$ is taken from [12, Table 6.23] (and $d_{0}^{*}$ follows from applying the relation discussed in Section 3.4, i.e., $d_{0}^{*}=d_{0} / 2-1$ ).

The proof goes along the same lines as that of Proposition 3.

Remark 1. Although we have presented Proposition 4 for general $k$, in practice we shall mostly use $k \in\{1,2\}$. For larger $k$, the restriction $D_{0}<D_{k} \leq\left(D_{0}\right)^{2}$ causes the conditions for $d_{0}(p) \geq D_{0}$ to dominate the search.

In Table 1 we show a list of primes for which $d_{k}(p)$ is strictly increasing, for $k \in\{1,2\}$. See also Figure 2, where we graphically compare our method to Yu's work in terms of the achieved comparison range.

\subsection{Finding Primes with High $d_{k}^{*}(p)$}

We can also apply the method from Section 4.3 to find $p$ with large $d_{k}^{*}(p)$, but the search procedure needs to be modified slightly. We present the appropriate modifications to the conditions.

Definition 6. Let $D_{0}, D_{k}$ be non-negative integers with $D_{0}<D_{k} \leq\left(D_{0}\right)^{2}$. Let $Q=\left\{q_{0}, \ldots, q_{k}\right\}$ be a set of $k+1$ distinct primes. We say that $Q$ is a $*$-related set on $\left(D_{0}, D_{k}\right]$ if $Q \subseteq\left(D_{0}, D_{k}\right]$ and there exist positive odd integers $x_{0}, \ldots, x_{k}<D_{0}$ such that:

1. for any $i$ with $0 \leq i \leq k$ we have $x_{i} q_{i} \leq D_{k}$

2. for any $i, j$ with $0 \leq i<j \leq k$ it holds that $\left|x_{i} q_{i}-x_{j} q_{j}\right| \in\{2,4,6, \ldots, 4 k\}$

Proposition 5. Let $D_{0}, D_{k}$ be non-negative integers with $D_{0}<D_{k} \leq\left(D_{0}\right)^{2}$, and let $p$ be a Blum prime with $d_{0}^{*}(p) \geq \frac{1}{2} D_{0}$. Then $d_{k}^{*}(p) \geq \frac{1}{2} D_{k}-k$ if and only if the following condition holds: for every set $Q$ of $k+1$ distinct primes $*$-related on $\left(D_{0}, D_{k}\right]$, it holds that there exists some $q \in Q$ with $(q \mid p)=1$. 
Table 1: Sequence of primes in increasing order (and their bit lengths $\ell$ ) for which $d_{k}(p)$ is strictly increasing, for $k \in\{1,2\}$. The primes below the dashed lines (for $\ell \geq 37$ ) have been found via our simplified search method, which means that there could exist smaller primes that give rise to the same or higher values of $d_{1}(p)$ resp. $d_{2}(p)$. The primes below " $\star \star \star$ " are the 64 bit primes with the highest $k$-range that we found.

\begin{tabular}{|c|c|c|}
\hline$\ell$ & $p$ & $d_{1}(p)$ \\
\hline 5 & 23 & 5 \\
\hline 5 & 31 & 10 \\
\hline 7 & 71 & 11 \\
\hline 8 & 167 & 13 \\
\hline 8 & 191 & 19 \\
\hline 10 & 599 & 20 \\
\hline 11 & 1319 & 37 \\
\hline 12 & 3119 & 40 \\
\hline 14 & 9719 & 45 \\
\hline 14 & 14951 & 60 \\
\hline 17 & 110039 & 65 \\
\hline 18 & 211559 & 66 \\
\hline 19 & 283631 & 67 \\
\hline 19 & 289511 & 72 \\
\hline 19 & 333791 & 109 \\
\hline 21 & 1884791 & 129 \\
\hline 22 & 2817239 & 140 \\
\hline 24 & 10522511 & 149 \\
\hline 25 & 25155191 & 156 \\
\hline 25 & 29036999 & 157 \\
\hline 27 & 79107311 & 179 \\
\hline 27 & 89658791 & 217 \\
\hline 30 & 927633671 & 227 \\
\hline 31 & 1514970551 & 276 \\
\hline 36 & 56709623759 & 277 \\
\hline 36 & 60221191631 & 281 \\
\hline 37 & 81720228911 & 291 \\
\hline 37 & 86345286719 & 339 \\
\hline 38 & 187800947879 & 396 \\
\hline 43 & 8714428081631 & 431 \\
\hline 44 & 10422103551551 & 437 \\
\hline 44 & 13729797542471 & 443 \\
\hline 47 & 78991232073599 & 452 \\
\hline 47 & 100395799811999 & 461 \\
\hline 54 & 12210981354571991 & 577 \\
\hline 54 & 13162388389217591 & 639 \\
\hline 57 & 93521022740468231 & 641 \\
\hline 57 & 141840650661890879 & 683 \\
\hline 60 & 692038256994017639 & 685 \\
\hline 61 & 1507647405383450231 & 756 \\
\hline 63 & 5831572531519229351 & 809 \\
\hline \multicolumn{2}{|c|}{6412367130975574671999} & 732 \\
\hline
\end{tabular}

\begin{tabular}{|c|c|c|}
\hline$\ell$ & $p$ & $d_{2}(p)$ \\
\hline 5 & 23 & 8 \\
\hline 5 & 31 & 10 \\
\hline 7 & 71 & 11 \\
\hline 8 & 167 & 14 \\
\hline 8 & 191 & 19 \\
\hline 8 & 239 & 20 \\
\hline 9 & 359 & 26 \\
\hline 9 & 479 & 35 \\
\hline 11 & 1151 & 38 \\
\hline 11 & 1511 & 41 \\
\hline 12 & 3527 & 43 \\
\hline 12 & 3911 & 58 \\
\hline 13 & 6551 & 59 \\
\hline 14 & 8951 & 66 \\
\hline 14 & 12239 & 89 \\
\hline 15 & 25679 & 140 \\
\hline 19 & 289511 & 176 \\
\hline 20 & 662639 & 182 \\
\hline 22 & 2798351 & 212 \\
\hline 24 & 10328111 & 223 \\
\hline 24 & 16178399 & 226 \\
\hline 25 & 17431391 & 250 \\
\hline 25 & 19632791 & 255 \\
\hline 25 & 25380911 & 276 \\
\hline 25 & 30809159 & 280 \\
\hline 26 & 53422151 & 290 \\
\hline 27 & 92989511 & 308 \\
\hline 28 & 246241511 & 318 \\
\hline 29 & 442696271 & 329 \\
\hline 30 & 721250351 & 379 \\
\hline 30 & 984093431 & 458 \\
\hline 35 & 18233703479 & 498 \\
\hline 35 & 29919732911 & 502 \\
\hline 37 & 95110047119 & 508 \\
\hline 38 & 149120083199 & 562 \\
\hline 38 & 241922449271 & 570 \\
\hline 40 & 696567525359 & 588 \\
\hline 40 & 700217963639 & 608 \\
\hline 41 & 1291095727151 & 640 \\
\hline 41 & 2088877265999 & 668 \\
\hline 43 & 8590297237079 & 720 \\
\hline 44 & 10268163904319 & 724 \\
\hline 45 & 18623040462311 & 743 \\
\hline 45 & 21505791039431 & 785 \\
\hline 46 & 49223854293071 & 878 \\
\hline 48 & 154272279972359 & 884 \\
\hline 52 & 3392073984672071 & 931 \\
\hline 55 & 21749977880115911 & 938 \\
\hline 56 & 38746340388406031 & 1046 \\
\hline 56 & 47155355205792599 & 1265 \\
\hline 60 & 984926255933291591 & 1283 \\
\hline 63 & 7143849267677035679 & 1336 \\
\hline \multicolumn{2}{|c|}{6413030782144247916831} & 1316 \\
\hline
\end{tabular}


Table 2: Sequence of primes in increasing order (and their bit lengths $\ell$ ) for which $d_{k}^{*}(p)$ is strictly increasing, for $k \in\{1,2\}$. Primes below the dashed lines have been found via our simplified search method, which means that there could exist smaller primes that give rise to the same or higher values of $d_{1}^{*}(p) \operatorname{resp.} d_{2}^{*}(p)$. For the primes above the dashed lines, it holds that the prime is the smallest

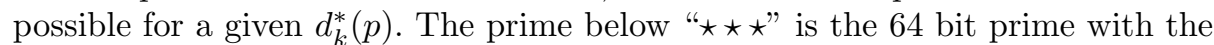
highest value of $d_{2}^{*}(p)$ that we found.

\begin{tabular}{|c|c|c|}
\hline$\ell$ & $p$ & $d_{1}^{*}(p)$ \\
\hline 5 & 23 & 1 \\
\hline 6 & 47 & 4 \\
\hline 7 & 83 & 5 \\
\hline 8 & 131 & 7 \\
\hline 8 & 239 & 8 \\
\hline 8 & 251 & 14 \\
\hline 10 & 1019 & 16 \\
\hline 11 & 1091 & 24 \\
\hline 13 & 4259 & 30 \\
\hline 14 & 10331 & 33 \\
\hline 14 & 12011 & 34 \\
\hline 17 & 74051 & 42 \\
\hline 17 & 96851 & 44 \\
\hline 19 & 420731 & 47 \\
\hline 20 & 831899 & 52 \\
\hline 20 & 878099 & 53 \\
\hline 20 & 954971 & 68 \\
\hline 23 & 5317259 & 78 \\
\hline 25 & 19127891 & 79 \\
\hline 25 & 31585979 & 94 \\
\hline 28 & 140258219 & 98 \\
\hline 30 & 697955579 & 104 \\
\hline 31 & 1452130811 & 112 \\
\hline 31 & 1919592419 & 115 \\
\hline 33 & 4323344819 & 116 \\
\hline 33 & 4499001491 & 117 \\
\hline 33 & 6024587819 & 118 \\
\hline 34 & 9259782419 & 138 \\
\hline 35 & 19846138451 & 143 \\
\hline 36 & 34613840351 & 151 \\
\hline 37 & 73773096179 & 153 \\
\hline 37 & 119607747731 & 174 \\
\hline 38 & 163030664579 & 182 \\
\hline 38 & 170361409391 & 207 \\
\hline 43 & 4754588149211 & 229 \\
\hline 48 & 171772053182831 & 242 \\
\hline 48 & 178774759690511 & 243 \\
\hline 48 & 205152197251811 & 258 \\
\hline 52 & 2950193919326891 & 259 \\
\hline 52 & 3705750905778011 & 284 \\
\hline 54 & 10624213337944379 & 296 \\
\hline 55 & 26259748609914431 & 321 \\
\hline 57 & 141840650661890879 & 340 \\
\hline 59 & 321961111376298371 & 345 \\
\hline 61 & 1158960903343074191 & 348 \\
\hline 61 & 1561357330831673339 & 378 \\
\hline 64 & 9409569905028393239 & 383 \\
\hline
\end{tabular}

\begin{tabular}{|c|c|c|}
\hline$\ell$ & $p$ & $d_{2}^{*}(p)$ \\
\hline 6 & 47 & 3 \\
\hline 7 & 83 & 6 \\
\hline 8 & 131 & 8 \\
\hline 8 & 179 & 15 \\
\hline 10 & 1019 & 16 \\
\hline 11 & 1091 & 26 \\
\hline 11 & 1427 & 31 \\
\hline 11 & 1811 & 36 \\
\hline 14 & 9539 & 51 \\
\hline 15 & 19211 & 68 \\
\hline 19 & 334619 & 78 \\
\hline 20 & 717419 & 80 \\
\hline 21 & 1204139 & 104 \\
\hline 22 & 2808251 & 114 \\
\hline 24 & 8774531 & 116 \\
\hline 24 & 11532611 & 117 \\
\hline 25 & 18225611 & 152 \\
\hline 27 & 98962211 & 155 \\
\hline 28 & 247330859 & 166 \\
\hline 30 & 738165419 & 174 \\
\hline 30 & 1030152059 & 188 \\
\hline 31 & 1456289579 & 197 \\
\hline 32 & 2451099251 & 206 \\
\hline 34 & 11159531291 & 207 \\
\hline 34 & 13730529419 & 216 \\
\hline 35 & 17221585499 & 219 \\
\hline 35 & 19186524419 & 232 \\
\hline 35 & 26203369331 & 242 \\
\hline 37 & 92830394411 & 248 \\
\hline 37 & 128808841619 & 287 \\
\hline 38 & 232481520059 & 324 \\
\hline 39 & 408727560491 & 335 \\
\hline 40 & 807183995411 & 370 \\
\hline 44 & 15869813229371 & 373 \\
\hline 45 & 19379613618119 & 411 \\
\hline 46 & 46760546950211 & 412 \\
\hline 48 & 240160967391791 & 425 \\
\hline 49 & 294269750529611 & 456 \\
\hline 53 & 8755197891979139 & 526 \\
\hline 57 & 85283169141238571 & 528 \\
\hline 58 & 148892345027857499 & 599 \\
\hline 61 & 1915368196138563011 & 648 \\
\hline 64 & $\begin{array}{c}\star \star \star \star \\
10807930853257193939\end{array}$ & 623 \\
\hline
\end{tabular}




\subsection{Implementation and Results}

We have implemented a search algorithm for primes $p$ with minimal $d_{k}(p)$ and $d_{k}^{*}(p)$ for $k=0,1,2$ using the precomputation of linear congruences as detailed above. We have enumerated all minimal $p$ up to 64 bits with ascending $d_{1}(p)$ and with $d_{0}(p) \geq 64$, and likewise for ascending $d_{1}^{*}(p)$ with $d_{0}^{*}(p) \geq 32$. Our implementation is written in Rust and uses the wheel method from [16]. It is publicly available on GitHub [1].

Table 2 shows results of our search for primes that give rise to as high as possible values of $d_{1}^{*}(p)$ and $d_{2}^{*}(p)$.

Open Problem. We leave open the problem of proving asymptotic lower bounds (in the Hardy-Littlewood's Big-Omega sense) as well as (better) upper bounds on $d_{k}(p)$ and $d_{k}^{*}(p)$ for $k \geq 1$.

\section{Secure Protocols for bsgn}

In this section we present several protocols for evaluating the bsgn function, assuming that $p$ is a Blum prime. Note that these protocols immediately imply comparison protocols; from the triangle inequality it follows that correctness for comparison is guaranteed if both inputs lie in $[-\lfloor d / 2\rfloor,\lfloor d / 2\rfloor]$, where $[-d, d]$ is the input range of the bsgn protocol.

We first present protocol Legendre, shown as Protocol 1, for securely evaluating the Legendre symbol. The protocol is stated in terms of black-box invocations of protocols RandomBit() for securely sampling a random bit $\{0,1\} \subset \mathbb{Z} / p \mathbb{Z}$ and RandomElem $\left.\left((\mathbb{Z} / p \mathbb{Z})^{*}\right)\right)$ for securely sampling a random element from $(\mathbb{Z} / p \mathbb{Z})^{*}$.

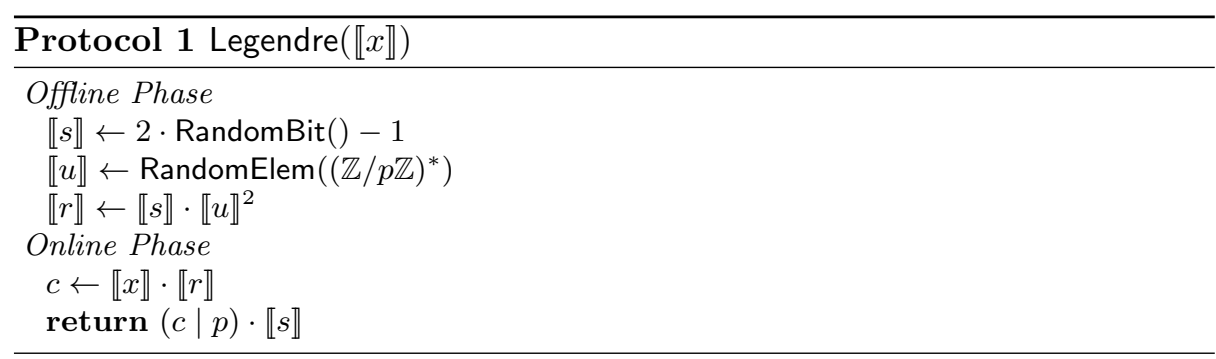

\subsection{Secure Medium-Range bsgn Protocol for $k=1$}

In our protocol for $k=1$, shown as Protocol 2, we compute the binary sign of the sum of the Legendre symbols by means of the multivariate polynomial

$$
f(x, y, z)=\frac{x+y+z-x y z}{2} .
$$

It is easy to verify that $f$ correctly computes the sign of the sum of $x, y, z \in$ $\{-1,+1\}$. The two secure multiplications required for the evaluation of $f(x, y, z)$ 
can be combined with the secure evaluations of the Legendre symbols such that the round complexity is not increased, as shown next.

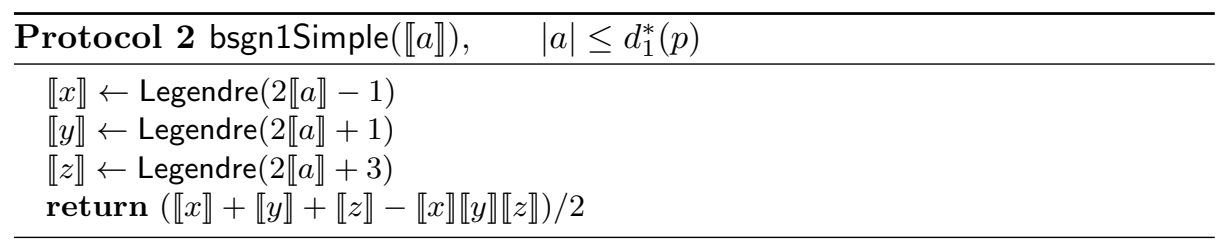

Decreasing the round complexity in the online phase. Protocol bsgn1Simple requires three rounds in the online phase. We can bring this down to a single round by precomputing the product of the random Legendre symbols produced in the offline phase of the Legendre protocol. This is shown in Protocol 3. The random bit protocol has been concretely instantiated in the offline phase of Protocol 3 to show that the product of the three random Legendre symbols can be computed in parallel to the preparation of their corresponding random elements. The offline phase requires two rounds in addition to the round complexity of securely sampling random elements of $(\mathbb{Z} / p \mathbb{Z})^{*}$.

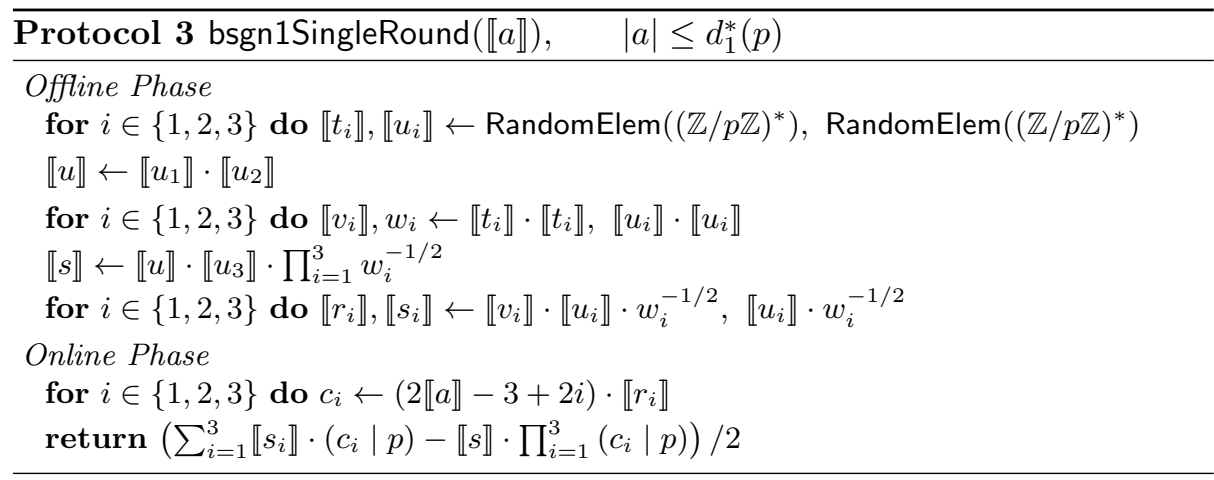

\subsection{Secure Medium-Range bsgn Protocol for $k=2$}

In our protocol for $k=2$, shown as Protocol 4, we compute the binary sign of the sum of the five Legendre symbols by means of another invocation of Legendre. In the latter (outer) invocation of Legendre, we need not apply the $x \mapsto 2 x+1$ map because we sum an odd number of values in $\{-1,+1\}$ which cannot become zero. Note that this requires that $d_{0}(p) \geq 5$ for correctness of the protocol.

Similar to the $k=1$ case, we may replace the evaluation of the Legendre symbol at the end by the evaluation of a suitable polynomial. For instance, one can use the univariate polynomial $f(x)=\left(3 x^{5}-110 x^{3}+1067 x\right) / 960$, which maps $x \in\{1,3,5\}$ to 1 and $x \in\{-1,-3,-5\}$ to -1 . This polynomial can be evaluated in three rounds using ordinary secure multiplication. Alternatively, a 5-variate 
polynomial can be used, in which case the required secure multiplications can be combined again with the secure evaluations of the Legendre symbols such that the round complexity of the online phase is not increased (as in the $k=1$ case).

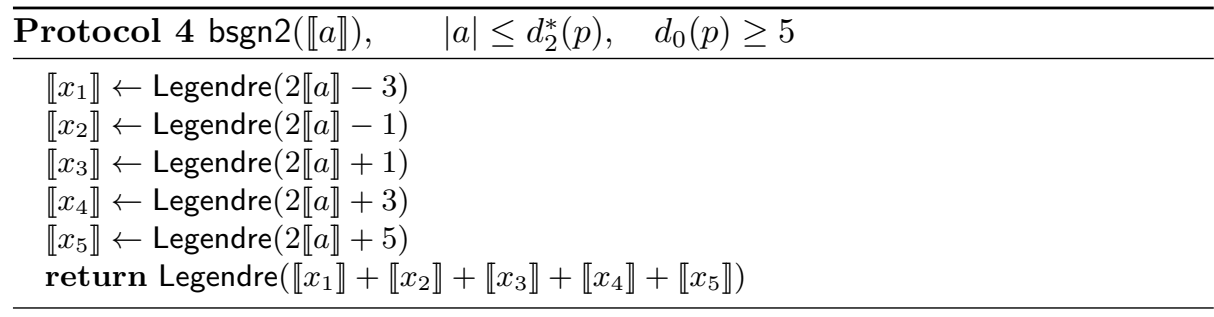

\section{Application: Fast Neural Network Evaluation in MPC}

In this section we demonstrate the usefulness of our secure binary-sign evaluation technique for securely evaluating a neural network.

\subsection{Binarized Multi-Layer Perceptron for MNIST}

For our experiments, we take the binarized multi-layer perceptron of Courbariaux et al. for recognizing handwritten digits from the well-known MNIST benchmark data set [7], which we refer as BMLP below. The BMLP network uses the sign function as its non-linear activation function, and is designed to be evaluated using integer arithmetic only. This allows for a natural MPC implementation.

The MNIST data set contains images of 28-by-28 pixels, where the intensity of each pixel is represented by a byte, i.e., an integer in $\mathcal{B}:=[0,255](0$ represents black, 255 represents white, and the values in between represent shades of gray). For the BMLP network, an input image is represented as a byte vector $\boldsymbol{x} \in \mathcal{B}^{784}$. Note that by reshaping a two-dimensional image into a (one-dimensional) vector the spatial structure is lost, but this is not a problem for multi-layer perceptrons (as opposed to convolutional neural networks, for instance).

Let $n$ denote the number of neurons per layer. The BMLP network consists of four layers, and uses $n=4096$. We view each layer $L_{i}, i \in[1,4]$, as a map between an input and output vector:

$$
\begin{aligned}
& L_{1}: \quad \mathcal{B}^{784} \rightarrow\{-1,+1\}^{n}, \\
& L_{i}:\{-1,+1\}^{n} \rightarrow\{-1,+1\}^{n}, \quad i \in\{2,3\} \\
& L_{4}:\{-1,+1\}^{n} \rightarrow \mathbb{Z}^{10} .
\end{aligned}
$$

Let $k_{1}=k_{2}=k_{3}=m_{2}=m_{3}=m_{4}=n$ and $k_{4}=10$ and $m_{1}=784$. In [7], the output of $L_{i}$ is computed as

$$
L_{i}(\boldsymbol{x}):= \begin{cases}\operatorname{BinarySign}\left(\operatorname{BatchNorm}_{\Theta_{i}}^{k_{i}}\left(W_{i} \boldsymbol{x}+\boldsymbol{b}_{i}\right)\right), & i \in\{1,2,3\} \\ \operatorname{BatchNorm}_{\Theta_{i}}^{k_{i}}\left(W_{i} \boldsymbol{x}+\boldsymbol{b}_{i}\right) & i=4 .\end{cases}
$$


Here $W_{i} \in\{-1,+1\}^{k_{i} \times m_{i}}$ is a matrix of weights, and $\boldsymbol{b}_{i} \in \mathbb{Z}^{k_{i}}$ is a vector of bias values. The function BatchNorm, which applies batch normalization elementwise, is defined as

$$
\begin{aligned}
\operatorname{BatchNorm}_{\Theta_{i}}^{\ell}: \mathbb{Z}^{\ell} & \rightarrow \mathbb{Z}^{\ell} \\
\left(x_{1}, \ldots, x_{\ell}\right) & \mapsto\left(f_{\Theta_{i}, 1}\left(x_{1}\right), \ldots, f_{\Theta_{i}, \ell}\left(x_{\ell}\right)\right)
\end{aligned}
$$

where $\Theta_{i}:=\left(\boldsymbol{\mu}_{i}, \tilde{\boldsymbol{\sigma}}_{i}, \boldsymbol{\gamma}_{i}, \boldsymbol{\beta}_{i}\right)$ are the batch norm parameters for the $i$ th layer: $\boldsymbol{\mu}_{i}=\left(\mu_{i, 1}, \ldots, \mu_{i, \ell}\right), \tilde{\boldsymbol{\sigma}}_{i}=\left(\tilde{\sigma}_{i, j}\right)_{j \in[1, \ell]}, \boldsymbol{\gamma}=\left(\gamma_{i, j}\right)_{j \in[1, \ell]}$, and $\boldsymbol{\beta}=\left(\beta_{i, j}\right)_{j \in[1, \ell]}$, and

$$
f_{\Theta_{i}, j}(x):=\gamma_{i, j}\left(\frac{x-\mu_{i, j}}{\tilde{\sigma}_{i, j}}\right)+\beta_{i, j} .
$$

The function BinarySign applies the bsgn function element-wise,

$$
\text { BinarySign : } \begin{aligned}
\mathbb{Z}^{n} & \rightarrow\{-1,+1\}^{n} \\
\left(x_{1}, \ldots, x_{n}\right) & \mapsto\left(\operatorname{bsgn}\left(x_{1}\right), \ldots, \operatorname{bsgn}\left(x_{n}\right)\right) .
\end{aligned}
$$

To obtain the final output of the BMLP, which is an integer $y \in[0,9]$, we apply an (oblivious) argmax operation to the output of $L_{4}$ :

$$
y:=\arg \max L_{4}\left(L_{3}\left(L_{2}\left(L_{1}(\boldsymbol{x})\right)\right)\right) \text {. }
$$

Training the Network. We have trained the BMLP on a GPU using Courbariaux' original implementation (described in [7]) which is publicly available on GitHub.

\subsection{Eliminating Redundant Parts of Batch Normalization}

In layers 1-3, the BinarySign function is applied directly to the output of the BatchNorm function. Because the bsgn function is invariant to multiplying its input by a positive scalar, the BatchNorm function might perform some operations that are immediately undone by the bsgn function. Indeed, it actually turns out that the BatchNorm function (when followed by the BinarySign function) reduces to an additional bias term; the authors of [7] seem to have overlooked this. Formally,

$$
\begin{aligned}
f_{i}(x) & =\gamma_{i}\left(\frac{x-\mu_{i}}{\tilde{\sigma}_{i}}\right)+\beta_{i}=\frac{\gamma_{i}}{\tilde{\sigma}_{i}}\left(x-\mu_{i}+\frac{\beta_{i} \tilde{\sigma}_{i}}{\gamma_{i}}\right), \\
\operatorname{bsgn}\left(f_{i}(x)\right) & =\operatorname{bsgn}\left(x-\mu_{i}+\frac{\beta_{i} \tilde{\sigma}_{i}}{\gamma_{i}}\right), \quad \gamma_{i}, \tilde{\sigma}_{i}>0 .
\end{aligned}
$$

Hence, we update the bias vector in all layers except the last as follows,

$$
\boldsymbol{b}_{i}^{\prime}:=\boldsymbol{b}_{i}-\boldsymbol{\mu}_{i}+\frac{\boldsymbol{\beta}_{i} \tilde{\boldsymbol{\sigma}}_{i}}{\gamma_{i}}
$$

where all operations (addition, subtraction, multiplication, and division) in the above expression are performed element-wise. With this modification, evaluation of the BMLP network simplifies to

$$
L_{i}(\boldsymbol{x})= \begin{cases}\operatorname{BinarySign}\left(W_{i} \boldsymbol{x}+\boldsymbol{b}_{i}^{\prime}\right), & i \in\{1,2,3\} \\ \operatorname{BatchNorm}_{\Theta_{i}}^{k_{i}}\left(W_{i} \boldsymbol{x}+\boldsymbol{b}_{i}\right) & i=4 .\end{cases}
$$




\subsection{Instantiating the BinarySign Function Per Layer}

Our aim is to instantiate the BinarySign function using our medium-range bsgn protocols. Nonetheless, for layer $L_{1}$, the magnitudes of the elements in the vector $W_{1} \boldsymbol{x}+\boldsymbol{b}_{1}^{\prime}$ for some image $\boldsymbol{x} \in \mathcal{B}^{784}$ will typically be way too large compared to the input range on which our bsgn protocols guarantee a correct answer. Hence, for $L_{1}$ we instantiate BinarySign as the element-wise application of an "off-theshelf" large-range bsgn protocol, such as Toft's comparison protocol [18].

For layers $L_{2}$ and $L_{3}$ we instantiate BinarySign with (element-wise applications of) Protocol bsgn1Simple using a 64-bit prime modulus $p$ for which $d_{1}^{*}(p)=383$, and, in separate experiments, with bsgn2 using a 64-bit modulus $p^{\prime}$ for which $d_{2}^{*}\left(p^{\prime}\right)=594$, and with Yu's method, using a 62-bit modulus $p^{\prime \prime}$ for which $d_{0}^{*}\left(p^{\prime \prime}\right)=134 .{ }^{6}$ Also for these layers, there seems to be a mismatch between the input ranges of bsgn1Simple, bsgn2 and Yu's method on which they guarantee correctness, i.e., $[-383,383],[-594,594]$ and $[-134,134]$ respectively, and the magnitudes of the elements in the vector $W_{i} \boldsymbol{y}+\boldsymbol{b}_{i}^{\prime}$ for $i \in\{2,3\}$, where $\boldsymbol{y} \in\{-1,+1\}^{n}$. The first term in this sum (the vector $W_{i} \boldsymbol{y}$ ), can have elements with magnitude equal to $n$ in the worst case, where $n=4096$. Nonetheless, the distribution of values in the vector $W_{i} \boldsymbol{y}+\boldsymbol{b}_{i}^{\prime}$ for all $i \in\{2,3\}$ is strongly concentrated around zero, hence we will just ignore the fact that our bsgn-protocols will be invoked a number of times on values outside the range for which they guarantee correctness. As we show quantitatively in Table 3, for our protocols this does not deteriorate the classification performance compared to a network where the full-range sign protocol is also used in layers $L_{2}$ and $L_{3}$, while for Yu's method (with prime $p^{\prime \prime}$ ) the error rate increases by $38 \%$. (Surprisingly, using bsgn1Simple even slightly improves the performance on the MNIST test set.)

\subsection{Experimental Results (Neural Network Evaluation)}

We have implemented the neural network in MPyC, a Python framework for secure multiparty computation [15]. The case $k=0$ comes down to applying the map $x \mapsto 2 x+1$ to the input followed by invoking Protocol 1 , for $k=1$ we used a mixture of Protocol 2 and Protocol 3, and for $k=2$ we used Protocol 4, in all cases expanding the calls to Protocol 1 to parallelize the secure computations of the Legendre symbol as much as possible. As a baseline we use the MPyC built-in secure comparison protocol, which is based on Toft's protocol [18]. For a meaningful performance evaluation, we set the bit length to 10 bits for the built-in comparisons used in layers 2 and 3 . We have also vectorized the code for all these comparison protocols, handling $n=4096$ comparisons at the same time for layers $1-3$, which increases the speed considerably.

We have run our experiments on a 3PC-LAN setup (CPUs: Intel four-core 4th generation Core i7 3.6 GHz). A complete evaluation between three parties on a secret-shared input image, using secret-shared weights and bias vectors,

\footnotetext{
${ }^{6}$ The prime moduli are $p=9409569905028393239, p^{\prime}=15569949805843283171$ and $p^{\prime \prime}=3546374752298322551$.
} 
Table 3: Classification performance of the BMLP on 10,000 MNIST test images

\begin{tabular}{lcccc}
\hline & Full-Range Sign & Yu $(k=0)$ & bsgn1Simple & bsgn2 \\
\hline Number of misclassifications & 248 & 342 & 227 & 247 \\
Error rate & 0.0248 & 0.0342 & 0.0227 & 0.0247 \\
\hline
\end{tabular}

runs in 59 seconds for $k=0,60$ seconds for $k=1$, in 62 seconds for $k=2$, and in 67 seconds for full-range comparisons. For evaluation of a batch of 10 input images the times are 205, 223, 235, and 302 seconds, respectively. The times for processing all comparisons in layers 2 and 3 are 6, 20,34, and 99 seconds, respectively. Hence, in this experiment the Legendre-based comparisons with $k=1$ are about 5 times faster than full-range comparisons. Similar speedups may be expected with other MPC frameworks for applications with comparisons restricted to medium-sized integers.

To determine the error rate for our particular BMLP, we have also implemented it in Python (including the Python counterparts of Yu's method and the Protocols bsgn1Simple and bsgn2, producing exactly the same errors outside their input ranges). The results measured for the 10,000 MNIST test images are shown in Table 3.

\section{Acknowledgments}

This work has received funding from the European Union's Horizon 2020 research and innovation program under grant agreements No 731583 (SODA) and No 780477 (PRIViLEDGE). We thank Frank Blom for running all our 3-party experiments on his $3 \mathrm{PC}-\mathrm{LAN}$ setup.

\section{References}

1. Abspoel, M.: Search for primes with high $d_{1}, d_{2}$ (2018), https://github.com/ abspoel/dk-search

2. Ankeny, N.C.: The least quadratic non residue. Ann. Math. 55(1), 65-72 (1952)

3. Feige, U., Kilian, J., Naor, M.: A minimal model for secure computation (extended abstract). In: Proceedings STOC '94. pp. 554-563 (1994)

4. Fridlender, V.R.: On the least $n$th power non-residue. Dokl. Akad. Nauk. SSSR 66, 351-352 (1949)

5. Graham, S.W., Ringrose, C.J.: Lower bounds for least quadratic non-residues. In: Berndt, B.C., et al. (eds.) Analytic number theory: Proceedings of a conference in honor of Paul T. Bateman. pp. 269-309. Boston, MA (1990)

6. Hildebrand, A.: On the least pair of consecutive quadratic nonresidues. Mich. Math. J. 34(1), 57-62 (1987)

7. Hubara, I., Courbariaux, M., Soudry, D., El-Yaniv, R., Bengio, Y.: Quantized neural networks: Training neural networks with low precision weights and activations. J. Mach. Learn. Res. 18(187), 1-30 (2018) 
8. Hudson, R.H.: The least pair of consecutive character non-residues. J. Reine Angew. Math. pp. 219-220 (1974)

9. Jacobsthal, E.: Anwendungen einer Formel aus der Theorie der quadratischen Reste. Ph.D. thesis, Friedrich-Wilhelms-Universität, Berlin, Germany (1906)

10. Lamzouri, Y., Li, X., Soundararajan, K.: Conditional bounds for the least quadratic non-residue and related problems. Math. Comput. 84(295), 2391-2412 (2015)

11. Linnik, U.V.: On the least prime in an arithmetic progression. I. The basic theorem. Rec. Math. [Mat. Sbornik] N.S. 15(57), 139-178 (1944)

12. Lukes, R.F.: A very fast electronic number sieve. Ph.D. thesis, University of Manitoba, Winnipeg, Canada (1995)

13. Pritchard, P.: A sublinear additive sieve for finding prime numbers. Commun. ACM 24(1), 18-23 (1981)

14. Salié, H.: Über den kleinsten positiven quadratischen Nichtrest nach einer Primzahl. Math. Nachr. 3(1), 7-8 (1949)

15. Schoenmakers, B.: MPyC - Secure multiparty computation in Python. GitHub (2018), v0.4.7, https://github.com/lschoe/mpyc

16. Sorenson, J.: The pseudosquares prime sieve. In: ANTS-VII, Germany, 2006. LNCS, vol. 4076, pp. 193-207. Springer (2006)

17. Sun, Z.H.: Consecutive numbers with the same Legendre symbol. Proc. Am. Math. Soc. 130(9), 2503-2507 (2002)

18. Toft, T.: Primitives and applications for multi-party computation. Ph.D. thesis, Aarhus Universitet, Denmark (2007)

19. Treviño, E.: The least $k$ th power non-residue. J. Number Theory 149, 201-224 (2015)

20. Xylouris, T.: Über die Nullstellen der Dirichletschen L-Funktionen und die kleinste Primzahl in einer arithmetischen Progression. Ph.D. thesis, Rheinischen FriedrichWilhelms-Universität Bonn, Germany (2011)

21. Yao, A.C.: Protocols for secure computations. In: 23rd Annual symposium FOCS '82. pp. 160-164 (1982)

22. Yu, C.H.: Sign modules in secure arithmetic circuits. Cryptology ePrint Archive, Report 2011/539 (2011), http://eprint.iacr.org/2011/539 